\title{
Quality of reporting in endoscopic ultrasound: Results of an international multicenter survey (the QUOREUS study)
}

\section{(ㄷ)(요 $\odot$}

\section{Authors}

Pietro Fusaroli ${ }^{1}$, Mohamad Eloubeidi ${ }^{2}$, Claudio Calvanese ${ }^{1}$, Christoph Dietrich ${ }^{3}$, Christian Jenssen ${ }^{4}$, Adrian Saftoiu $^{5}$, Claudio De Angelis ${ }^{6}$, Shyam Varadarajulu ${ }^{7}$, Bertrand Napoleon ${ }^{8}$, Andrea Lisotti ${ }^{1}$, the QUOREUS study group

Institutions

1 Gastroenterology Unit, Hospital of Imola, Department of Medical and Surgical Sciences - DIMEC, University of Bologna, Italy

2 Anniston digestive health, Anniston, Alabama, United States

3 Department of Internal Medicine 2, Caritas Krankenhaus, Bad Mergentheim, Germany

4 Department of Internal Medicine, Krankenhaus Märkisch Oderland Strausberg/Wriezen; Brandenburg Institute of Clinical Ultrasound, Medical University Brandenburg, Neuruppin, Germany

5 Department of Gastroenterology, Research Center of Gastroenterology and Hepatology, University of Medicine and Pharmacy of Craiova, Craiova, Romania

6 Department of General and Specialist Medicine, Gastroenterologia-U, Città della Salute e della Scienza di Torino, Turin, Italy

7 Center for Interventional Endoscopy, AdventHealth Orlando, Orlando, Florida, USA.

8 Digestive Endoscopy Unit, Hopital Privé J Mermoz Ramsay Générale de Santé, Lyon, France

submitted 16.1.2021

accepted after revision 18.3 .2021

\section{Bibliography}

Endosc Int Open 2021; 09: E1171-E1177

DOI 10.1055/a-1482-7769

ISSN 2364-3722

(c) 2021. The Author(s).

This is an open access article published by Thieme under the terms of the Creative Commons Attribution-NonDerivative-NonCommercial License, permitting copying and reproduction so long as the original work is given appropriate credit. Contents may not be used for commercial purposes, or adapted, remixed, transformed or built upon. (https://creativecommons.org/licenses/by-nc-nd/4.0/)

Georg Thieme Verlag KG, Rüdigerstraße 14,

70469 Stuttgart, Germany

Corresponding author

Prof. Pietro Fusaroli, Gastroenterology Unit, Hospital of Imola, Department of Medical and Surgical Science, University of Bologna, Italy, Via Montericco 4, 40026 Imola (BO), Italy
Fax: +390542662409

pietro.fusaroli@unibo.it

\# Supplementary material is available under https://doi.org/10.1055/a-1482-7769

\section{ABSTRACT}

Background and study aims The endoscopic report has a key role in quality improvement for gastrointestinal endoscopy. High quality standards have been set by the endoscopic societies in this field. Unlike other digestive endoscopy procedures, the quality of reporting in endoscopic ultrasound (EUS) has not been thoroughly evaluated and a reference standard is lacking.

Methods We performed an international online survey concerning the attitudes of endosonographers towards EUS reports in order to understand the needs for standardization and quality improvement. Endosonographers from different countries and institutional setting, with varying case volume and experience were invited to take part to complete a structured questionnaire.

Results We collected replies from 171 endosonographers. Overall analysis of results according to case volume, experience and working environment of respondents (academic, public hospital, private) are provided. In brief, everyone agreed on the need for standardization of EUS reporting. The use of minimal standard terminology and a structured tree with mandatory items was considered of primary importance. Image documentation was also deemed fundamental in complementing EUS reports both for patient documentation and research purposes. A strong demand for connection and consultation among endosonographers for clinical and training needs was also found. In this respect, a formal expert consultation network was advocated in order to improve the quality of reporting in EUS.

Conclusions Our survey showed a strong agreement among endosonographers who expressed the need for a standardization in order to improve the report and, as a consequence, the quality of EUS. 


\section{Introduction}

A well-written report is the most important way of communication between clinicians, referring doctors and patients. Appropriate construction, clarity, and clinical focus of medical documentation are essential to high-quality patient care.

Reports play a key role for quality improvement in digestive endoscopy, too [1]. All endoscopic procedures should be systematically documented and completely legible in endoscopic reports [2,3]. In this regard, endoscopic scientific societies have issued recommendations for standardizing reports and overcoming the wide variability that still exists. Indeed, heterogeneity has been recognized as the main source of incompleteness and mistakes in reporting [4-6].

A good endoscopic report should rely upon minimal standard terminology (MST) [6-8], structured trees, and image documentation for each procedure [9]. In this respect, electronic health records for endoscopy are very helpful. Current softwares allow saving text with mandatory fields, images and videos into a database that can always be available for medical, statistical, research and legal purposes [10-12].

Unlike digestive endoscopy, the quality of reporting in endoscopic ultrasound (EUS) has not been thoroughly evaluated and a reference standard is lacking [13-17]. EUS is a complex technique with several diagnostic and therapeutic applications [1824]. Due to this heterogeneity, it is essential to share validated criteria and items for EUS reporting.

We performed an international online survey to assess the routine of endosonographers in EUS reports and understand the needs for standardization and quality improvement in this particular field.

\section{Methods}

\section{Study design}

Physicians involved in EUS were invited to take part to a webbased survey and complete an online questionnaire. The invitation was sent by email to all physicians affiliated with the European Group for Endoscopic Ultrasonography (EGEUS). Recipients were asked to forward the invitation to colleagues who may have not been reached by the first invitation. The survey remained accessible online from July 2017 to July 2019. Trainees and gastroenterologists during post-graduate school were excluded.

After acceptance, the participants received a link to a dedicated online questionnaire. Survey responses were anonymously recorded by the internet site Surveymonkey.com, which allows qualitative and descriptive analysis.

\section{Questionnaire}

After requests for general information about the geographic area, institutional setting and EUS experience, the questionnaire consisted of 25 itemized questions subdivided into four sections: 1 writing EUS reports (7 questions); 2 EUS iconographic documentation (7 questions); 3 quality improvement of EUS reports (7 questions); and 4 EUS report structure (4 questions). A final blank field was available for suggestions.

\section{Statistical analysis}

All qualitative results were expressed as number and percentage. Subgroup analysis was done according to institutional setting, namely academic institution (AI), public hospital (PH), and private practice (PP), operator's experience $(\leq 5$ or $>5$ years) and yearly examination volume $(\leq 500$ or $>500$ procedures). For the comparisons between groups we used either Fisher exact test $(2 \times 2)$ or Chi-squared test when appropriate. $P<0.05$ was considered statistically significant. We used MedCalc package for Windows (version 19) for statistical analysis.

\section{Results}

\section{General information}

Surveys were sent to all physicians practicing EUS known by the authors; recipients were also asked to contribute to further spreading of the questionnaire to other endosonographers. Invaluable support was also given by the European Groups for Endoscopic Ultrasonography (EGEUS), which distributed the questionnaire to the member societies. After exclusion of incomplete responses, 171 questionnaires came back available for analysis. Most of the respondents were from Europe (75\%), the remainders were from Asia (13\%), North America (6\%), South America (5\%) and Africa (1\%).

Among all the physicians who responded, $36 \%$ were employed in academic institutions, $45 \%$ in public hospitals and $19 \%$ in private practice. Subgroup analysis according to institutional settings are reported in Supplementary Table $\mathbf{1}$.

The majority of participants (77\%) performed $\leq 500$ EUS procedures per year and $23 \%$ performed $>500$ procedures per year (Supplementary Table 2). Most of the respondents were experienced in EUS; in detail, 73 \% had been performing EUS for more than 5 years (Supplementary Table 3).

In detail, physicians working in $\mathrm{Al}$ and $\mathrm{PH}$ reported greater personal experience and yearly volume of examinations than those in PP $(P<0.001$ and $P<0.001$, respectively); similarly, yearly volumes of examinations per center were higher in $\mathrm{Al}$ and $\mathrm{PH}$ than $\mathrm{PP}(P=0.002)$. Moreover, respondents with $>5$ years of experience reported higher number of yearly procedures both per operator and per center $(P<0.001)$.

The annual volume of examinations was equally distributed between centers performing more than 500 procedures (47\%) and those performing less or equal to 500 procedures (53\%). The number of endosonographers was homogeneous among centers with just one operator (21\%), two operators (37\%), three operators ( $21 \%)$, and more than three operators (21\%).

In the following sections only the most relevant results are reported, which is why figures may not always round up to $100 \%$. Complete results are reported in > Table 1 and Supplementary Tables.

\section{Section 1: Writing EUS reports}

Most of physicians (85\%) typed the report by themselves while $10 \%$ used a dictation system (5\% gave other answers). Dictation was more common in PP as opposed to $\mathrm{Al}$ and $\mathrm{PH}$ (25\% vs. $3 \%$ and $9 \%$, respectively; $P=0.005)$. The majority of operators 
- Table 1 Complete results of QUOREUS study (respondents, $\mathrm{n}=171$ ).

\section{General information}

Please tell us your institutional setting:

- Academic institution

$62(36.3 \%)$

- Public hospital

$77(45.0 \%)$

- Private practice

$32(18.7 \%)$

How many EUS procedures do you perform personally

- $\leq 500$ exams

$132(77.2 \%)$

per year?

. $>500$ exams

$39(22.8 \%)$

How many years have you been performing EUS?

- $\leq 5$ years

$47(27.5 \%)$

- $>5$ years

$124(72.5 \%)$

How many EUS procedures are performed at your center?

- $\leq 500$ exams

$91(53.2 \%)$

. $>500$ exams

$80(46.8 \%)$

How many endosonographers are there in your center?

-

. 2

$36(21.1 \%)$

- 3

$64(37.4 \%)$

$+2$

$36(21.1 \%)$

- $>3$

$35(20.5 \%)$

\section{Writing EUS reports}

Q1-1: Do you personally write the report?

- Yes, I type it

$145(84.8 \%)$

- No, I dictate it

$17(9.9 \%)$

- Other answers

$9(5.3 \%)$

Q1-2: When do you take care of the report?

- At the end of each procedure

$158(92.4 \%)$

- At the end of EUS session

$11(6.4 \%)$

- On another day

$2(1.2 \%)$

Q1-3: Does the patient receive a report after the exam?

- Yes, before leaving the hospital

$105(61.4 \%)$

- Yes, but on a different day

$14(8.2 \%)$

- Yes, and a copy is mailed only to the referring physician too

$25(14.6 \%)$

- No, it is mailed only to the referring physician

$27(15.8 \%)$

Q1-4: Do you talk to the patient to provide additional explanations?

- Yes, always

$138(80.7 \%)$

- Yes, but only if requested

$32(18.7 \%)$

- No

$1(0.6 \%)$

Q1-4.1: Do you preferentially do it:

- On the same day

$158(93.5 \%)$

- On a different day

$11(6.5 \%)$

Q1-5: Do you use an Electronic Health Record to write your report?

Q1-6: Is your report visible by others via LAN or Web?

- Yes

$129(75.4 \%)$

- No $42(24.6 \%)$

- Yes, from all doctors in my unit $28(16.4 \%)$

- Yes, from all doctors in my hospital $103(60.2 \%)$

- Yes, from all doctors in my regional area $17(9.9 \%)$

- Yes, from all doctors in my Country $3(1.8 \%)$

- No $27(15.8 \%)$

used to write the report at the end of each procedure (92\%) and just a few of them at the end of the session (6\%); only $1 \%$ declared writing the report on another day.
The EUS report was given to the patients in $84 \%$ of cases, and a copy was also mailed to the referring physicians in $15 \%$ of cases; however, in $16 \%$ of cases the report was only mailed to the referring physicians. While $80 \%$ of respondents stated 
- Table 1 (Continuation)

\section{EUS iconographic documentation}

Q2-1': Do you save still pictures of the exam?

Q2-2: Do you routinely record videos of the exam?

Q2-2.1 ${ }^{1}$ : If you save videos, in which format?

Q2-3: Does the patient receive images of the exam along with the text?

Q2-4: Does the patient receive a video of the exam?

Q2-4.1: In which format?

Q2-5: Do you think it would be important to provide a video to the patient in the future?

\section{Improving quality of reporting}

Q3-1: Do you review the video of the examination before writing the report?

Q3-2: Do you think that reviewing the videos of EUS examination routinely before writing the report could be useful?

Q3-2.1: What prevents you from doing it?

3-3: Do you look for consultation with colleagues from your center before writing the report?

Q3-4: Do you look for consultation with colleagues from another center before writing the report?

Q3-4.1: How often do you do it per year?

- Yes, print images

$68(39.8 \%)$

- Yes, digital images

$124(72.5 \%)$

- No

$5(2.9 \%)$

- Yes, I record the whole procedure

$19(11.1 \%)$

- Yes, I record just selected parts

$33(19.3 \%)$

- No, but I record the interesting cases

$87(50.9 \%)$

- No, I do not record videos

$33(19.3 \%)$

- PC video files (MPEG, AVI, etc.)

$123(71.9 \%)$

- DVD/digital recorder

$50(29.2 \%)$

- Analogic recorder

$9(5.3 \%)$

- Yes, with figure legends

$51(29.8 \%)$

- Yes, without figure legends

$56(32.7 \%)$

- No, he receives just the text

$65(38.0 \%)$

- Yes

$2(1.2 \%)$

- Only upon request

$34(19.9 \%)$

- No

$135(78.9 \%)$

- CD

$12(33.3 \%)$

- DVD

$19(52.8 \%)$

- USB key

$13(36.1 \%)$

- Yes

- No

$68(50.4 \%)$

$67(49.6 \%)$

- Yes, always

$22(12.9 \%)$

- Only in selected classes

$88(51.5 \%)$

- No

$61(35.7 \%)$

- Yes, it could improve diagnostic and/or staging accuracy

$72(48.3 \%)$

- No

$78(52.3 \%)$

- Lack of time

$87(58.8 \%)$

- Lack of time

$26(17.6 \%)$

- I do not record videos

$42(28.4 \%)$

- Yes, routinely

$9(5.3 \%)$

- Yes, but only in difficult cases

$92(54.1 \%)$

- No

$69(40.6 \%)$

- Yes, I send pictures by email

$21(12.4 \%)$

- Yes, I send videos by email

$11(6.5 \%)$

- Yes, I bring personally the documentation to the colleague

$11(6.5 \%)$

- No

$127(74.7 \%)$

- 1-3 cases

$24(55.8 \%)$

- 4-10 cases

$15(34.9 \%)$

- $>10$ cases 
- Table 1 (Continuation)

Q3-5: Do you think that a formally organized consultation system could be useful?

\section{EUS report structure}

Q4-1: Do you write the report according to a structured tree?

Q4-2: Do you think that a minimal standard terminology could be useful?

Q4-3: Do you think that a structured report with compulsory items should be used in EUS?

Q4-41: Which of the following items are included in your report?
- Yes, it should be provided by scientific societies

$63(37.1 \%)$

- Yes, it should be provided by EUS experts on an individual basis

- No, it is enough to have it on a personal relationship
- Yes, I describe both normal and abnormal findings

- Yes, but I describe only abnormal findings

- No, my report changes on an individual basis

- Yes, and it should appear on the report

- Yes, but it should be used for statistics and should not appear on the report

- No

- Yes, it should be quite detailed and it should be different according to indication

- Yes, but it should be a generic format to be used as a reference

- No, each one should write the report according to personal or institutional routine

- Indication

- Type of sedation

- Type of instrument

- Echoendoscope reprocessing data

- Descriptive report

- Conclusive remarks

- TNM staging

- Advice for EUS follow-up

- Nurses name

- Vital parameters

- FNA or therapeutic procedure

- Complications

- Non-EUS Follow-up

- Referring physician
$138(81.2 \%)$

$12(7.1 \%)$

$20(11.8 \%)$

$150(88.2 \%)$

$13(7.6 \%)$

$126(74.1 \%)$

$33(19.4 \%)$

$11(6.5 \%)$

$152(89.4 \%)$

$153(90.0 \%)$

$152(89.4 \%)$

$47(27.6 \%)$

$168(98.8 \%)$

$167(98.2 \%)$

$157(92.4 \%)$

$149(87.6 \%)$

$84(49.4 \%)$

$45(26.5 \%)$

$16(9.4 \%)$

$5(2.9 \%)$

$9(5.3 \%)$

$3(1.8 \%)$

EUS, endoscopic ultrasound; FNA, fine-needle aspiration

${ }^{1}$ Multiple answers allowed.

that they always gave oral explanations to patients together with the report, $19 \%$ used to do it only upon request.

Three out of four clinicians who responded utilized electronic health records, which were more frequently available in $\mathrm{Al}$ and $\mathrm{PH}$ compared to $\mathrm{PP}$ (74\% and $82 \%$ vs. $63 \%$, respectively; $P$ $<0.001)$. In most cases the report was accessible to other doctors via Local Area Network or Web from the same unit/hospital (77\%), the regional area (10\%) or the entire Country (2\%).

\section{Section 2: EUS iconographic documentation}

EUS pictures were stored in a digital format by $73 \%$ of endosonographers; in $40 \%$ of cases images were thermally printed, while in $3 \%$ of cases reports consisted in text only. Storing digi- tal images was more common in Al vs. $\mathrm{PH}$ and $\mathrm{PP}$ (81\% vs. $70 \%$ and $63 \%$, respectively; $P=0.03$ ). Moreover, endosonographers with greater than 5-year experience were more used to store digital images than those with shorter experience (78\% vs. 57\%; $P=0.03$ ).

EUS video recording was not available to $19 \%$ of all respondents; endosonographers with $\leq 500$ exams per year were significantly less likely to record videos ( $23 \%$ vs. $7.7 \%$; $P=0.004$ ). However, video recording was significantly more common in Al than $\mathrm{PH}$ and $\mathrm{PP}(92 \%$ vs. $74 \%$ and $75 \%$, respectively; $P=$ 0.005).

Pictures of the EUS examination were attached to the report in $62 \%$ of cases, either with or without figure legends. In gener- 
al, a video of the EUS examination was not provided to the patients; however, it was available upon request in $20 \%$ of cases. As far as projects for the future were regarded, endosonographers with $>5$ years of experience perceived less important to provide a video to the patients than those with $\leq 5$ years of experience ( $55 \%$ vs. $36 \%$; $P=0.05$ ). A similar divergence was seen between respondents from $\mathrm{Al}$ vs. $\mathrm{PH}$ and $\mathrm{PP}$ ( $68 \%$ vs. $35 \%$ and $52 \%$, respectively; $P=0.003$ ).

\section{Section 3: Quality improvement of EUS reports}

Most of the respondents (59\%) were used to review the examination videos before writing the report; of these, $54 \%$ did so only in difficult cases but not routinely. Physicians working in $\mathrm{PH}$ reported a greater inclination to review videos than those in $\mathrm{Al}$ and $\mathrm{PP}$ ( $69 \%$ vs. $61 \%$ and $59 \%$, respectively; $P<0.001)$.

Nevertheless, half of the participants declared that a routine review of the EUS video before writing the report could be useful to improve diagnostic and staging accuracy but they were not allowed to do so due to lack of time. Interestingly, doctors working in PP would be more willing to review videos than those in $\mathrm{PH}$ and $\mathrm{Al}(67 \%$ vs. $49 \%$ and $38 \%$, respectively; $P=$ $0.04)$. Moreover, doctors with $\leq 5$ years of experience were more in favor of video review than those with greater experience ( $78 \%$ vs. $37 \%$; $P<0.001$ ) as well as those with $\leq 500$ examinations per year compared to those with $>500$ examinations (57\% vs. $20 \%$; $P<0.001$ ).

Participants sought consultation with a colleague from the same center in $59 \%$ of cases and with a colleague from another center (e.g. former mentor, renowned expert) in $25 \%$ of cases. Consultation with a colleague from another center was reported more frequently by respondents with inferior experience and procedure volume $(P=0.002$ and $P=0.02$, respectively). Most endosonographers ( $81 \%$ ) agreed that a formally organized consultation system could be helpful in this field.

\section{Section 4: EUS report structure}

Most endosonographers used a structured tree in the report describing either normal and abnormal findings (81\%) or only abnormal findings (7\%). Most were in favor of adopting MST either in the report ( $88 \%$ ) or for database purpose only ( $8 \%$ ).

While a structured report with or without different compulsory items according to the indication was deemed useful by $74 \%$ and $19 \%$ of respondents, respectively, only $7 \%$ preferred writing their reports based on personal or institutional routine.

\section{Discussion}

We reported the contributions from 171 endosonographers who responded to our online survey concerning the current quality of EUS report. Overall, we gathered valuable information regarding writing and sharing of reports, handling of photo and video documentation, management of difficult cases, structuring of mandatory items and MST trees, and suggestions for improvement. Moreover, we found some disparities in the attitude towards using digital resources, reviewing videos and seeking online consultation among physicians from different institutional settings such as AI, PH and PP. Differences were also noted among physicians with different personal case volume and/or years of experience.

The endoscopic report has a key role in quality improvement of gastrointestinal endoscopy. Our scientific societies have provided suggestions to overcome the existing variability, which is the main cause of incompleteness and mistakes. A proper report should be based on MST and a standardized structure of the text and image documentation for each procedure. Electronic health records offer structured reports with mandatory items in order to fulfill all the requirements for setting up a proper patient documentation and storing their data for any future purpose.

Unlike other endoscopic procedures, the quality of reporting in EUS has not been adequately investigated. More than 20 years ago, Aabakken wrote a seminal contribution entitled "Standardized terminology in endoscopic ultrasound" to implement an MST for EUS maintaining that the need for standardization in this field was even more important than in general digestive endoscopy [25]. However, little interest has followed since then. The French society of digestive endoscopy provides formats of EUS report on their internet site (www.sfed.org) that are available only in French and deal only with esophageal and rectal cancer, pancreatic and ampullary cancer, and submucosal tumors.

Our survey provides a snapshot of the current practice in EUS reports around the world. Respondents provided information that is useful to assess the points of strength and weakness of what is already done. Moreover, many suggestions proved useful for future improvement of the quality of EUS report. Scientific societies might be interested in obtaining such information for the benefit of patient care, physician education and scientific research.

It was common belief among our respondents that future EUS reports shall be more structured by adopting mandatory items, structured trees and MST. Moreover, endosonographers with minor experience and case volume wished that more time was available to carefully review the videos of their examinations before writing a report and also to make the videos available to patients. If this practice was implemented, it would imply at least partially rethinking the current routine of our busy endoscopic practices.

In addition, many endosonographers believe that consultation with EUS experts is fundamental to better understand difficult cases before writing a report. In theory, formal consultation could be integrated not only in mentorship programs but also in peer coaching, which is becoming increasingly popular [26].

In our view, the reason for standardizing EUS reports is at least threefold. First, EUS should provide reliable and verifiable information about disease diagnosis and staging, which should come in a readable and reproducible format useful for subsequent care and therapeutic management also by physicians of different specialties. Second, communication among endosonographers from different centers is possible only if patients' EUS reports are always understandable and contain complete information. This might prove essential in particular when EUS-guided fine needle aspiration is repeated at another center 
in case of inconclusive or negative previous findings [27]. Third, research and further development of the technique are made possible when the information provided is consistent and correlated with appropriate image documentation as is already the case for radiological procedures.

\section{Conclusions}

In conclusion, based on our survey, there is strong agreement among endosonographers on the need for standardization of EUS reports using structured trees and MST. Still images should always be saved in a digital database and become part of the report; video recordings are necessary in selected cases. There is a need for networking and consultation among endosonographers for clinical and educational purposes. In this respect, a formally organized international consultation system would facilitate communication among endosonographers in order to improve the quality of reporting. We are aware that many efforts are needed before standards of reporting in EUS become validated and internationally established. Nevertheless, we believe we have provided a starting point for future studies in view of such a standardization.

\section{Competing interests}

The authors declare that they have no conflict of interest.

\section{References}

[1] Rizk MK, Sawhney MS, Cohen J et al. Quality indicators common to all Gl endoscopic procedures. Gastrointest Endosc 2015; 81: 3-16

[2] Tang S], Raju G. Endoscopic photography and image documentation. Gastrointest Endosc 2015; 82: 925-931

[3] Marques S, Bispo M, Pimentel-Nunes P et al. Image documentation in gastrointestinal endoscopy: review of recommendations. GE Port J Gastroenterol 2017; 24: 269-274

[4] Maratka Z. Terminology, definitions and diagnostic criteria in digestive endoscopy. With the collaboration of the members of the Terminology Committee of the World Society of Digestive Endoscopy/ OMED. Scand J Gastroenterol Suppl 1984; 103: 1-74

[5] American Society for Gastrointestinal Endoscopy. Quality improvement of gastrointestinal endoscopy: guidelines for clinical application. From the ASGE. Gastrointest Endosc 1999; 49: 842-844

[6] ESGE Committee for Minimal Standards for Terminology and Documentation in Digestive Endoscopy. Minimal standard terminology for databases in digestive endoscopy. Bad Homburg, Germany: NORMED Verlag; 1995

[7] Aabakken L, Rembacken B, LeMoine O et al. Minimal standard terminology for gastrointestinal endoscopy -MST 3.0. Endoscopy 2009; 41: $727-728$

[8] Korman LY, Delvaux M, Crespi M. The minimal standard terminology in digestive endoscopy: perspective on a standard endoscopic vocabulary. Gastrointest Endosc 2001; 53: 392-396

[9] Aabakken L, Barkun AN, Cotton PB et al. Standardized endoscopic reporting. J Gastroenterol Hepatol 2014; 29: 234-240
[10] Armstrong D, Barkun A, Bridges R et al. Canadian Association of Gastroenterology Safety and Quality Indicators in Endoscopy Consensus Group. Canadian Association of Gastroenterology consensus guidelines on safety and quality indicators in endoscopy. Can J Gastroenterol 2012; 26: 17-31

[11] Beaulieu D, Barkun AN, Dubé C et al. Endoscopy reporting standards. Can J Gastroenterol 2013; 27: 286-292

[12] Bretthauer M, Aabakken L, Dekker E et al. ESGE Quality Improvement Committee. Requirements and standards facilitating quality improvement for reporting systems in gastrointestinal endoscopy: European Society of Gastrointestinal Endoscopy (ESGE) Position Statement. Endoscopy 2016; 48: 291-294

[13] Schwab R, Pahk E, Lachter J. Impact of endoscopic ultrasound quality assessment on improving endoscopic ultrasound reports and procedures. World J Gastrointest Endosc 2016; 8: 362-367

[14] Lachter J, Bluen B, Waxman I et al. Establishing a quality indicator format for endoscopic ultrasound. World J Gastrointest Endosc 2013; 5: $574-580$

[15] Jacobson BC, Chak A, Hoffman B et al. ASGE/ACG Taskforce on Quality in Endoscopy. Quality indicators for endoscopic ultrasonography. Am J Gastroenterol 2006; 101: 898-901

[16] Fusaroli P, Kypreos D, Petrini CA et al. Scientific publications in endoscopic ultrasonography: Changing trends in the third millennium. J Clin Gastroenterol 2011; 45: 400-404

[17] Fusaroli P, Kypraios D, Eloubeidi MA et al. Levels of evidence in endoscopic ultrasonography: A systematic review. Digest Dis Sci 2012; 57 : 602-609

[18] Coe SG, Raimondo M, Woodward TA et al. Quality in EUS: an assessment of baseline compliance and performance improvement by using the American Society for Gastrointestinal Endoscopy-American College of Gastroenterology quality indicators. Gastrointest Endosc 2009; 69: 195-201

[19] Cassani L, Aihara H, Anand GS et al. American Society for Gastrointestinal Endoscopy Training Committee. Core curriculum for EUS. Gastrointest Endosc 2020; 92: 469-473

[20] Jenssen C, Hocke M, Fusaroli P et al. EFSUMB Guidelines on Interventional Ultrasound (INVUS), Part IV - EUS-guided interventions: General Aspects and EUS-guided Sampling (Short Version). Ultraschall in der Medizin 2016; 37: 157-169

[21] Fusaroli P, Jenssen C, Hocke M et al. EFSUMB Guidelines on Interventional Ultrasound (INVUS), Part V. Ultraschall in der Medizin 2016; 37: E77-E99

[22] Domagk D, Oppong KW, Aabakken L et al. Performance measures for ERCP and endoscopic ultrasound: a European Society of Gastrointestinal Endoscopy (ESGE) Quality Improvement Initiative. Endoscopy 2018; 50: 1116-1127

[23] Facciorusso A, Buccino RV, Muscatiello N. How to measure quality in endoscopic ultrasound. Ann Transl Med 2018; 6: 266

[24] Wani S, Wallace MB, Cohen J et al. Quality indicators for EUS. Gastrointest Endosc 2015; 81: 67-80

[25] Aabakken L. Standardized terminology in endoscopic ultrasound. Eur ] Ultrasound 1999; 10: 179-183

[26] Kumar NL, Housiaux A, Ryou M. How to continue learning after gastroenterology fellowship with a peer-coach. Gastroenterology 2020; 158: 812-815

[27] Lisotti A, Frazzoni L, Fuccio L et al. Repeat EUS-FNA of pancreatic masses after nondiagnostic or inconclusive results: systematic review and meta-analysis. Gastrointest Endosc 2020; 91: 1234-1241 